\title{
Team Papelo: Transformer Networks at FEVER
}

\author{
Christopher Malon \\ NEC Laboratories America \\ malon@nec-labs.com
}

\begin{abstract}
We develop a system for the FEVER fact extraction and verification challenge that uses a high precision entailment classifier based on transformer networks pretrained with language modeling, to classify a broad set of potential evidence. The precision of the entailment classifier allows us to enhance recall by considering every statement from several articles to decide upon each claim. We include not only the articles best matching the claim text by TFIDF score, but read additional articles whose titles match named entities and capitalized expressions occurring in the claim text. The entailment module evaluates potential evidence one statement at a time, together with the title of the page the evidence came from (providing a hint about possible pronoun antecedents). In preliminary evaluation, the system achieves .5736 FEVER score, .6108 label accuracy, and .6485 evidence $F 1$ on the FEVER shared task test set.
\end{abstract}

\section{Introduction}

The release of the FEVER fact extraction and verification dataset (Thorne et al., 2018) provides a large-scale challenge that tests a combination of retrieval and textual entailment capabilities. To verify a claim in the dataset as supported, refuted, or undecided, a system must retrieve relevant articles and sentences from Wikipedia. Then it must decide whether each of those sentences, or some combination of them, entails or refutes the claim, which is an entailment problem. Systems are evaluated on the accuracy of the claim predictions, with credit only given when correct evidence is submitted.

As entailment data, premises in FEVER data differ substantially from those in the image caption data used as the basis for the Stanford Natural Language Inference (SNLI) (Bowman et al., 2015) dataset. Sentences are longer (31 compared to 14 words on average), vocabulary is more abstract, and the prevalence of named entities and out-ofvocabulary terms is higher.

The retrieval aspect of FEVER is not straightforward either. A claim may have small word overlap with the relevant evidence, especially if the claim is refuted by the evidence.

Our approach to FEVER is to fix the most obvious shortcomings of the baseline approaches to retrieval and entailment, and to train a sharp entailment classifier that can be used to filter a broad set of retrieved potential evidence. For the entailment classifier we compare Decomposable Attention (Parikh et al., 2016; Gardner et al., 2017) as implemented in the official baseline, ESIM (Chen et al., 2017), and a transformer network with pre-trained weights (Radford et al., 2018). The transformer network naturally supports out-of-vocabulary words and gives substantially higher performance than the other methods.

\section{Transformer network}

The core of our system is an entailment module based on a transformer network. Transformer networks (Vaswani et al., 2017) are deep networks applied to sequential input data, with each layer implementing multiple heads of scaled dot product attention. This attention mechanism allows deep features to be compared across positions in the input.

Many entailment networks have two sequence inputs, but the transformer is designed with just one. A separator token divides the premise from the hypothesis.

We use a specific transformer network released by OpenAI (Radford et al., 2018) that has been pre-trained for language modeling. The network consists of twelve blocks. Each block consists of a 
multi-head masked self-attention layer, layer normalization (Ba et al., 2016), a feed forward network, and another layer normalization. After the twelfth block, two branches exist. In one branch, matrix multiplication and softmax layers are applied at the terminal sequence position to predict the entailment classification. In the other branch, a hidden state is multiplied by each token embedding and a softmax is taken to predict the next token. The language modeling branch has been pre-trained on the BookCorpus dataset (Zhu et al., 2015). We take the pre-trained model and train both branches on examples from FEVER.

\section{Reframing entailment}

The baseline FEVER system (Thorne et al., 2018) ran the AllenNLP (Gardner et al., 2017) implementation of Decomposable Attention (Parikh et al., 2016) to classify a group of five premise statements concatenated together against the claim. These five premise statements were fixed by the retrieval module and not considered individually. In our system, premise statements are individually evaluated.

We collect training data as the five sentences with the highest TFIDF score against the claim, taken from the Wikipedia pages selected by the retrieval module. If any ground truth evidence group for a claim requires more than one sentence, the claim is dropped from the training set. Otherwise, each sentence is labeled with the truth value of the claim if it is in the ground truth evidence set, and labeled as neutral if not. The resulting data forms an entailment problem that we call "FEVER One." For comparison, we form "FEVER Five" and "FEVER Five Oracle" by concatenating all five retrieved sentences, as in the baseline. In FEVER Five Oracle, the ground truth is the claim ground truth (if verifiable), but in FEVER Five, ground truth depends on whether the retrieved evidence is in the ground truth evidence set.

Several FEVER claims require multiple statements as evidence in order to be supported or refuted. The number of such claims is relatively small: in the first half of the development set, only 623 of 9999 claims were verifiable and had no singleton evidence groups. Furthermore, we disagreed with many of these annotations and thought that less evidence should have sufficed. Thus we chose not to develop a strategy for multiple evidence statements.
To compare results on FEVER Five to FEVER One, we must aggregate decisions about individual sentences of possible evidence to a decision about the claim. We do this by applying the following rules:

1. If any piece of evidence supports the claim, we classify the claim as supported.

2. If any piece of evidence refutes the claim, but no piece of evidence supports it, we classify the claim as refuted.

3. If no piece of evidence supports or refutes the claim, we classify the claim as not having enough information.

We resolve conflicts between supporting and refuting information in favor of the supporting information, because we observed cases in the development data where information was retrieved for different entities with the same name. For example, Ann Richards appeared both as a governor of Texas and as an Australian actress. Information that would be a contradiction regarding the actress should not stop evidence that would support a claim about the politician.

Even if a sentence is in the evidence set, it might not be possible for the classifier to correctly determine whether it supports the claim, because the sentence could have pronouns with antecedents outside the given sentence. Ideally, a coreference resolution system could add this information to the sentence, but running one could be time consuming and introduce its own errors. As a cheap alternative, we make the classifier aware of the title of the Wikipedia page. We convert any undersores in the page title to spaces, and insert the title between brackets before the rest of each premise sentence. The dataset constructed in this way is called "FEVER Title One."

The FEVER baseline system works by solving FEVER Five Oracle. Using Decomposable Attention, it achieves .505 accuracy on the test half of the development set. Swapping in the Enhanced Sequential Inference Model (ESIM) (Chen et al., 2017) to solve FEVER Five Oracle results in an accuracy of .561. Because ESIM uses a single out-of-vocabulary (OOV) token for all unknown words, we expect it to confuse named entities. Thus we extend the model by allocating 10,000 indices for out-of-vocabulary words with randomly initialized embeddings, and taking a hash of each 


\begin{tabular}{lcccc}
\hline Problem & \multicolumn{2}{c}{ Support } & \multicolumn{2}{c}{ Claim } \\
& Accuracy & Kappa & Accuracy & Kappa \\
\hline ESIM on FEVER One & .760 & .260 & .517 & .297 \\
ESIM on FEVER Title One & .846 & .394 & .639 & .433 \\
Transformer on FEVER Title One & .958 & .660 & .823 & .622 \\
\hline
\end{tabular}

Table 1: Effect of adding titles to premises.

\begin{tabular}{lcccc}
\hline Problem & \multicolumn{2}{c}{ Support } & \multicolumn{2}{c}{ Claim } \\
& Accuracy & Kappa & Accuracy & Kappa \\
\hline ESIM on FEVER Title Five Oracle & N/A & N/A & .591 & .388 \\
ESIM on FEVER Title Five & N/A & N/A & .573 & .110 \\
ESIM on FEVER Title One & .846 & .394 & .639 & .433 \\
Transformer on FEVER Title Five Oracle & N/A & N/A & .673 & .511 \\
Transformer on FEVER Title Five & N/A & N/A & .801 & .609 \\
Transformer on FEVER Title One & .958 & .660 & .823 & .622 \\
\hline
\end{tabular}

Table 2: Concatenating evidence or not.

\begin{tabular}{lc}
\hline System & Retrieval \\
\hline FEVER Baseline (TFIDF) & $66.1 \%$ \\
+ Titles in TFIDF & $68.3 \%$ \\
+ Titles + NE & $80.8 \%$ \\
+ Titles + NE + Film & $81.2 \%$ \\
Entire Articles + NE + Film & $90.1 \%$ \\
\hline
\end{tabular}

Table 3: Percentage of evidence retrieved from first half of development set. Single-evidence claims only.

\begin{tabular}{lcc}
\hline System & Development & Test \\
\hline FEVER Title Five Oracle & .5289 & - \\
FEVER Title Five & .5553 & - \\
FEVER Title One & .5617 & .5539 \\
FEVER Title One (Narrow Evidence) & .5550 & - \\
FEVER Title One (Entire Articles) & .5844 & .5736 \\
\hline
\end{tabular}

Table 4: FEVER Score of various systems. All use NE+Film retrieval.

OOV word to select one of these indices. With extended ESIM, the accuracy is .586. Therefore, we run most later comparisons with extended ESIM or transformer networks as the entailment module, rather than Decomposable Attention.

The FEVER One dataset is highly unbalanced in favor of neutral statements, so that the majority class baseline would achieve $93.0 \%$ on this data. In fact it makes training ESIM a challenge, as the model only learns the trivial majority class predictor if the natural training distribution is followed. We reweight the examples in FEVER One for ESIM so that each class contributes to the loss equally. Then, we use Cohen's Kappa rather than the accuracy to evaluate a model's quality, so that following the bias with purely random agreement is not rewarded in the evaluation. In Table 1 we compare FEVER One to FEVER Title One, both at the level of classifying individual support statements and of classifying the claim by aggregating these decisions as described above. On a support basis, we find a $52 \%$ increase in Kappa by adding the titles.

When ESIM is replaced by the transformer network, class reweighting is not necessary. The network naturally learns to perform in excess of the majority class baseline. Cohen's Kappa is 68\% higher than that for ESIM.

The possibility of training on oracle labels for a concatenated set of evidence allows a classi- 
fier to simply guess whether the hypothesis is true and supported somewhere, rather than having to consider the relationship between hypothesis and premise. For example, it is possible to classify $67 \%$ of SNLI examples correctly without reading the premise (Gururangan et al., 2018). As we show in Table 2, for ESIM, we find that this kind of guessing makes the FEVER Title Five Oracle performance better than FEVER Title Five. The Transformer model is accurate enough that oracle guessing does not help. Both models perform best when classifying each bit of evidence separately and then aggregating.

\section{Improving retrieval}

Regardless of how strong the entailment classifier is, FEVER score is limited by whether the document and sentence retrieval modules, which produce the input to the entailment classifier, find the right evidence. In Table 3, we examine the percentage of claims for which correct evidence is retrieved, before filtering with the entailment classifier. For this calculation, we skip any claim with an evidence group with multiple statements, and count a claim as succesfully retrieved if it is not verifiable or if the statement in one of the evidence groups is retrieved. The baseline system retrieves the five articles with the highest TFIDF score, and then extracts the five sentences from that collection with the highest TFIDF score against the claim. It achieves $66.1 \%$ evidence retrieval.

Our first modification simply adds the title to each premise statement when computing its TFIDF against the claim, so that statements from a relevant article get credit even if the subject is not repeated. This raises evidence retrieval to $68.3 \%$.

A more significant boost comes from retrieving additional Wikipedia pages based on named entity recognition (NER). We start with phrases tagged as named entities by SpaCy (Honnibal and Johnson, 2015), but these tags are not very reliable, so we include various capitalized phrases. We retrieve Wikipedia pages whose title exactly matches one of these phrases.

The named entity retrieval strategy boosts the evidence retrieval rate to $80.8 \%$, while less than doubling the processing time. However, sometimes the named entity page thus retrieved is only a Wikipedia disambiguation page with no useful information. Noticing a lot of questions about films in the development set, we modify the strategy to also retrieve a page titled " $\mathrm{X}$ (film)" if it exists, whenever " $\mathrm{X}$ " is retrieved. The film retrievals raise evidence retrieval to $81.2 \%$.

Finally, we eliminate the TFIDF sentence ranking to expand sentence retrieval from five sentences to entire articles, up to the first fifty sentences from each. Thus we obtain 2.6 million statements to classify regarding the 19,998 claims in the shared task development set, for an average of 128 premises per claim. The evidence retrieval rate, including all these premises, increases to $90.1 \%$. We continue to apply the entailment module trained with only five premise retrievals. Running the entailment module on this batch using a machine with three NVIDIA GeForce GTX 1080Ti GPU cards takes on the order of six hours.

Retrieving more than five sentences means that we can no longer submit all retrieved evidence as support for the claims. Instead, we follow the aggregation strategy from Section 3 to decide the claim label, and only submit statements whose classification matches. Limiting evidence in this way when only five statements are retrieved ("narrow evidence" in Table 4) pushes FEVER score down very little, to .5550 from .5617 on the development set, so we have confidence that the extra retrieval will make up for the loss. Indeed, when the system reviews the extra evidence, FEVER score goes up to .5844 on the development set.

Table 4 compares the end-to-end performance of systems that evaluate five retrieved statements together, evaluate five retrieved statements separately, and evaluate all statements from entire articles separately. Evaluating the statements separately gives better performance. We submit the systems that retrieve five statements and entire articles for evaluation on the test set, achieving preliminary FEVER scores of .5539 and .5736 respectively (label accuracy of .5754 and .6108, evidence recall of .6245 and .5002 , evidence F1 of .2542 and .6485). In preliminary standings, the latter system ranks fourth in FEVER score and first in evidence F1.

\section{Discussion}

Our approach to FEVER involves a minimum of heuristics and relies mainly on the strength of the Transformer Network based entailment classification. The main performance gains come from adding retrievals that resolve named entities rather than matching the claim text only, filtering fewer 
of the retrievals, and making the entailment classifier somewhat aware of the topic of what it is reading by including the title. If higher quality and more plentiful multi-evidence claims would be constructed, it would be nice to incorporate dynamic retrievals into the system, allowing the classifier to decide that it needs more information about keywords it encountered during reading.

\section{References}

Lei Jimmy Ba, Ryan Kiros, and Geoffrey E. Hinton. 2016. Layer normalization. CoRR, abs/1607.06450.

Samuel Bowman, Gabor Angeli, Christopher Potts, and Christopher Manning. 2015. A large annotated corpus for learning natural language inference. In Proceedings of the Conference on Empirical Methods in Natural Language processing (EMNLP).

Qian Chen, Xiaodan Zhu, Zhen-Hua Ling, Si Wei, Hui Jiang, and Diana Inkpen. 2017. Enhanced LSTM for natural language inference. In Proceedings of the 55th Annual Meeting of the Association for Computational Linguistics (Volume 1: Long Papers), pages 1657-1668.

Matt Gardner, Joel Grus, Mark Neumann, Oyvind Tafjord, Pradeep Dasigi, Nelson F. Liu, Matthew Peters, Michael Schmitz, and Luke S. Zettlemoyer. 2017. AllenNLP: A deep semantic natural language processing platform. CoRR, abs/1803.07640.

Suchin Gururangan, Swabha Swayamdipta, Omer Levy, Roy Schwartz, Samuel Bowman, and Noah A. Smith. 2018. Annotation artifacts in natural language inference data. In Proceedings of the Conference of the North American Chapter of the Association for Computational Linguistics: Human Language Technologies (NAACL-HLT), Volume 2, pages 107-112.

Matthew Honnibal and Mark Johnson. 2015. An improved non-monotonic transition system for dependency parsing. In Proceedings of the Conference on Empirical Methods in Natural Language Processing (EMNLP), pages 1373-1378.

Ankur Parikh, Oscar Täckström, Dipanjan Das, and Jakob Uszkoreit. 2016. A decomposable attention model for natural language inference. In Proceedings of the Conference on Empirical Methods in Natural Language Processing (EMNLP), pages 22492255 .

Alec Radford, Karthik Narasimhan, Tim Salimans, and Ilya Sutskever. 2018. Improving language understanding by generative pre-training. In OpenAI Blog.

James Thorne, Andreas Vlachos, Christos Christodoulopoulos, and Arpit Mittal. 2018. FEVER: a large-scale dataset for fact extraction and verification. In Proceedings of the Conference of the North American Chapter of the Association for Computational Linguistics: Human Language Technologies (NAACL-HLT), Volume 1.

Ashish Vaswani, Noam Shazeer, Niki Parmar, Jakob Uszkoreit, Llion Jones, Aidan N Gomez, Ł ukasz Kaiser, and Illia Polosukhin. 2017. Attention is all you need. In Advances in Neural Information Processing Systems 30, pages 5998-6008.

Yukun Zhu, Ryan Kiros, Richard S. Zemel, Ruslan Salakhutdinov, Raquel Urtasun, Antonio Torralba, and Sanja Fidler. 2015. Aligning books and movies: Towards story-like visual explanations by watching movies and reading books. In Proceedings of the IEEE International Conference on Computer Vision (ICCV), pages 19-27. 